\title{
Drug Susceptibility Testing of Mycobacteria Isolated from Humans and Cattle from Selected Sites of Ethiopia
}

\author{
Mekonnen Tadesse $^{1 *}$, Gobena Ameni' ${ }^{2}$, Kassu Desta ${ }^{3}$ \\ ${ }^{1}$ Mailman School of Public Health-ICAP in Ethiopia, Columbia University, Addis Ababa, Ethiopia \\ ${ }^{2}$ Aklilu Lemma Institute of Pathobiology, Addis Ababa University, Addis Ababa, Ethiopia \\ ${ }^{3}$ Department of Medical Laboratory Sciences, Addis Ababa University, Addis Ababa, Ethiopia \\ Email: mt2758@columbia.edu, ${ }^{*}$ mektad2003@yahoo.com
}

Received 27 May 2014; revised 30 June 2014; accepted 12 July 2014

Copyright (C) 2014 by authors and Scientific Research Publishing Inc.

This work is licensed under the Creative Commons Attribution International License (CC BY). http://creativecommons.org/licenses/by/4.0/

(c) (i) Open Access

\section{Abstract}

Background: The effectiveness of a standard anti-tuberculosis (TB) treatment regimen correlates with in vitro drug susceptibility pattern of the infecting tubercle bacilli. The results of the drug susceptibility tests help select a proper treatment regimen or modify treatment regimen for a better management of patients and surveillance and timely control of the spread of the drug resistant TB in the community. Treatment of drug resistant TB is costly, and the outcomes, including survivorship, can be poor. As the result, the drug susceptibility test has become more important than ever. Objective: To determine the drug-susceptibility pattern of M. tuberculosis and M. bovis isolated from selected sites of Ethiopia. Methods: The conventional indirect Löwenstein-Jensen (L-J) proportion method was used to detect the drug susceptibility pattern of 29 isolates of $M$. $\mathbf{t u}$ berculosis and 21 isolates of $M$. bovis to four anti-TB drugs (streptomycin, rifampicin, isoniazid and ethambutol). Results: Resistance was observed only in M. tuberculosis isolates while all isolates of $M$. bovis were fully susceptible to the four drugs. Thus, the overall resistance of $M$. tubeculosis isolates to any of the four drugs was $\mathbf{5 1 . 7 \%}$. As such, any type of drug resistance was most frequent to streptomycin $(41.3 \%)$ followed by isoniazid $(20.6 \%)$ while it was minimal to rifampicin $(6.8 \%)$ and ethambutol (3.4\%). Multidrug resistant TB (MDR-TB) was not detected in the study. Conclusion: This preliminary study showed high level of resistance in $M$. tuberculosis isolates warranting appropriate use of anti-TB drugs in those sites from where the isolates were obtained.

\section{Keywords}

M. tuberculosis, M. bovis, Drug Resistance, Tuberculosis

\footnotetext{
${ }^{*}$ Corresponding author.
}

How to cite this paper: Tadesse, M., Ameni, G. and Desta, K. (2014) Drug Susceptibility Testing of Mycobacteria Isolated from Humans and Cattle from Selected Sites of Ethiopia. Journal of Tuberculosis Research, 2, 125-131. 


\section{Introduction}

The treatment of tuberculosis (TB) has been threatened by the increasing number of patients with drug resistant TB [1]. The most significant emergence has been that of the multi-drug resistant (MDR) strains, where single isolate is resistant to rifampicin and isoniazid, the two most potent anti-TB drugs. The treatment of MDR strains is prolonged and expensive with toxic second line drugs [2] [3].

Drug resistance has reached alarming levels with the emergence of strains that are virtually untreatable with existing drugs. MDR TB strains have emerged in all regions of the world and in Africa may be more prevalent than previously appreciated [4].

In Ethiopia, resistance to individual first-line anti-TB drugs is increasing. A study conducted a decade ago in the country revealed the overall prevalence of resistance to one or more anti-TB drugs to be 37.3\%. Primary and acquired resistance was 32.5\% and 51.2\% respectively. MDR was detected in 3.5\% of cases who had previous history of treatment [5]. But another study in 2001 indicated that over all resistance to one or more of the antiTB drugs was 19.5\% [6] and in 2008, Desta et al. reported no MDR TB but 13.5\% of polyresistant (resistant to two or more drugs other than simultaneous resistance to rifampicin and isoniazid) strains from smear negatives $[7]$.

Human TB of animal origin caused by Mycobacterium bovis (M. bovis) is becoming increasingly important in developing countries. In Sub-Saharan Africa, humans and animals share the same microenvironment and water holes, especially during droughts and the dry season, thereby, potentially promoting the transmission of $M$. bovis from animals to humans. It is zoonotic, being transmitted to humans by an aerogenous route and/or through consumption of infected milk and other cattle products [8] [9]. It has been estimated that M. bovis accounts globally for $3.1 \%$ of all human TB cases (2.1\% of all pulmonary and $9.4 \%$ of all extra-pulmonary tuberculosis (EPTB) cases) [10].

In Ethiopia, there is increasing evidence that $M$. bovis infections may be much more significant than generally considered [11]. Several studies conducted in Ethiopia have confirmed the transmission of M. tuberculosis and M. bovis between humans and cattle [12]-[15].

MDR strains of $M$. bovis have been associated with human cases in European countries with genotypes matching both medical and veterinary isolates. For example, Guerrero et al. reported the nosocomial transmission of $M$. bovis resistant to eleven drugs in people with advanced human immunodeficiency virus-1 (HIV-1) infection in Spain [16]-[18]. Patients infected with MDR TB strains not only pose a threat to themselves but also to the community as well [19]. Although the endemic nature of bovine tuberculosis (BTB) and the risk of human infection have been known for long, the pattern and extent of drug susceptibility of the etiologic agent of this human threat largely remained undetermined. Therefore, evaluation of drug susceptibility of M. bovis strains is important, not only for estimating such risk but also to understand the effect of uncontrolled use of antibiotics and the magnitude of influence of the transmission of drug resistant or MDR $M$. bovis strains on treatment of human TB [20], necessitating continued periodic monitoring of $M$. bovis isolates for development of antimicrobial resistance [16] [17].

The effectiveness of a standard anti-TB treatment regimen correlates with in vitro drug susceptibility pattern of the infecting tubercle bacilli. The results of the drug susceptibility tests help select a proper treatment regimen or modify treatment regimen for a better management of patients and surveillance and timely control of the spread of the drug resistant TB in the community. Treatment of drug resistant TB is costly, and the outcomes, including survivorship, can be poor. As the result, drug susceptibility test has become more important than ever [21]-[24].

In Ethiopia, although a little information is available on the drug susceptibility patterns of M. tuberculosis strains, there is no information on drug susceptibility pattern of $M$. bovis strains. Therefore, the purpose of this study was to evaluate the drug susceptibility patterns of M. tuberculosis and M. bovis strains isolated from selected sites of Ethiopia.

\section{Materials and Methods}

\subsection{Study Site and Isolates}

We hypothesize that drug resistant $M$. tuberculosis and $M$. bovis are prevalent in the study sites. To prove or disprove this hypothesis, a study was conducted on Mycobacterial species previously isolated from humans and 
cattle, characterized and appropriately preserved in the mycobacteriology laboratory of Aklilu Lemma Institute of Pathobiology, Addis Ababa University. The M. bovis isolates were obtained from cattle tissue samples collected from Kombolcha abattoir, northeastern Ethiopia; while M. tuberculosis isolates were recovered from sputum samples of patients who became smear positive from Sellale (Central Ethiopia), Wolaita (Southern Ethiopia) and Nekemte (Western Ethiopia). The patients from Sellale comprise both new and re-treatment cases; while cases from Wolaita and Nekemte are new cases only. At the time of the study, the patients were on DOTS chemotherapy as per the national guideline at local hospital and district health centers. Acid fast (Ziehl-Neelsen) staining [25] was done for all of the study isolates to check for purity of the isolates. The specific number of isolates obtained from different sites is shown in Table 1.

\subsection{The Drugs}

First line anti-TB drugs including, ethambutol, isoniazid, rifampicin, and streptomycin, all from Sigma Chemicals Co., Germany, were used in this study. Stock solutions for all the four drugs were prepared as per the standard procedure. Stock solutions of isoniazid, streptomycin, and ethambutol were prepared in sterile distilled water (SDW), whereas rifampicin was prepared in ethanol and methanol. Working solution of isoniazid was prepared by further diluting it with SDW. All the three stock solution and working solution of isoniazid were sterilized by filtering through $0.22 \mu \mathrm{m}$ membrane filter. The first $20 \%$ of the filtered solution were discarded, as most of the filters may initially retain some of the drug. The drug solutions were added to Lownestein-Jensen (L-J) media to give a critical concentration $(\mu \mathrm{g} / \mathrm{ml})$ of: streptomycin $4 \mu \mathrm{g} / \mathrm{ml}$, isoniazid $0.2 \mu \mathrm{g} / \mathrm{ml}$, rifampicin 40 $\mu \mathrm{g} / \mathrm{ml}$, and ethambutol $2 \mu \mathrm{g} / \mathrm{ml}$.

\subsection{Drug Susceptibility Testing}

Isolates that were frozen were thawed at room temperature. Three drops were inoculated onto a slant of L-J medium to get a primary culture. All $M$. bovis isolates were inoculated on pyruvate containing media, whereas $M$. tuberculosis on glycerol containing media and incubated at $37^{\circ} \mathrm{C}$ temperature and examined every week for bacterial growth. To prepare test inoculum, a loop full of Mycobacteria from the culture was taken and emulsified in a test tube containing $1 \mathrm{ml}$ of SDW and $6-7$ glass beads. About $4 \mathrm{ml}$ of SDW was added to it and allowed to stand for the coarse particles to settle down. The Mycobacterium solution was carefully decanted to other clear, sterile test tubes, and the opacity/turbidity was matched with McFarland standard no. 1 and adjusted by addition of SDW drop by drop. This bacterial suspension was estimated to contain $10^{6}$ to $10^{8} \mathrm{CFU} / \mathrm{ml}$. Two dilutions $\left(10^{-2}\right.$ and $\left.10^{-4}\right)$ were prepared from this bacterial suspension. A loop full of each dilution was inoculated to drug containing and drug free L-J media in test tubes. All the samples were incubated at $37^{\circ} \mathrm{C}$ and examined every week for growth.

On the twenty eighth and forty second days, test results were recorded by counting the number of colonies grown on each of drug containing and drug-free medium. Simultaneously, colony counts were recorded on a chart prepared particularly for this purpose. No further reading of the test was made for those strains classified as resistant on the twenty eighth day. A second reading was made on the forty second day for those strains whose result was sensitive at the twenty eighth day. The final definitive results for all the four drugs were reported on forty second day. The proportion of resistant bacilli was calculated by dividing the number of colonies obtained on drug containing medium with the number of colonies on drug free medium. Counting was made when the number of colonies on drug free was between fifty and one hundred and fifty so that the number of colonies is countable. The critical proportion of resistant bacilli required to define a strain as a resistant was $1 \%$ for each of the 4 drugs. A bacterial growth of more than $1 \%$ was taken as resistant. The proportion of bacteria less than $1 \%$ was considered as susceptible [26] [27].

Table 1. Distribution of the Mycobacteria species by site.
\begin{tabular}{ccc}
\hline Site & Species & Number (\%) \\
\hline Wolaita & M. tuberculosis & $9(18 \%)$ \\
Kombolcha & M. bovis & $21(42 \%)$ \\
Sellale & M. tuberculosis & $19(38 \%)$ \\
Nekemte & M. tuberculosis & $1(2.0 \%)$ \\
Total & & $50(100 \%)$ \\
\hline
\end{tabular}




\subsection{Quality Control}

All the recommended standard procedure [26] [27] were obeyed. The color of the media was checked before using it for culture, and only those media with appropriate color (light green) were chosen. Prior to inoculation all the media prepared in a day were incubated at $37^{\circ} \mathrm{C}$ for 48 hours to check for any contamination. H37Rv $M$. tuberculosis was used in each batch of drug susceptibility test for internal quality control purpose.

\section{Result}

This report is the first report on study of drug susceptibility of $M$. bovis in the country. Drug resistance patterns of the study isolates is presented in Table 2.

No drug resistance was observed for $M$. bovis. All the resistant isolates were $M$. tuberculosis. The overall resistance of $M$. tuberculosis to any of the tested drugs was $51.7 \%$, but the rest $48.3 \%$ were fully susceptible to the drugs. Resistance to only one drug was observed in $34.4 \%$ of the isolates, whereas resistance to any of the two drugs was detected in $13.7 \%$ of the study isolates. Resistance to any of the three drugs was detected in $3.4 \%$ of the isolates.

Any type of drug resistance was most frequent to streptomycin (41.3\%), followed by isoniazid (20.6\%), while resistance was minimal to rifampicin (6.8\%) and ethambutol (3.4\%). Mono-resistance to streptomycin, ethambutol, rifampicin and isoniazid is $24.1 \%, 0 \%, 3.4 \%$, and $6.8 \%$ respectively.

Combined drug resistance among study isolates was obtained more frequently to streptomycin and isoniazid (10.3\%) than to any combination of all other drugs. A single isolate resistant to streptomycin and rifampicin was also observed in this study. Triple resistance to streptomycin, ethambutol and isoniazid was $3.4 \%$. Resistance to all the four first-line anti-TB drugs and MDR-TB was not observed in the study.

M. tuberculosis isolates obtained from Sellale showed high rate of any type of drug resistance (41.4\%) than isolates from Wolaita Sodo (10.3\%). One isolate that was obtained from Nekemte was susceptible to all anti-TB drugs tested.

\section{Discussion}

The overall resistance rate of $M$. tuberculosis to one or more anti-TB drugs of $51.7 \%$ found in the present study, is similar with the previous report in the country, 51.2\% [5]. This result shows that there is high rate of resistance in M. tuberculosis strains to any of the four first-line anti-TB drugs in the sites from which the study isolates were obtained.

The resistance rate recorded for streptomycin (41.3\%) in M. tuberculosis isolates in the present study, is comparable with the report from Bangladesh, 55.65\% [28]. On the other hand, it is higher than the report from Arsi zone (11.4\% in primary resistance and 10.5\% in acquired resistances) [6] and Addis Ababa, 24.3\% [7]. As shown in Table 2, most of the streptomycin resistant isolates were from Sellale area. This drug has been in use

Table 2. Drug resistance patterns of M. tuberculosis isolates.

\begin{tabular}{|c|c|c|c|c|}
\hline & Mycobaterial Species & & Origin Sites & \\
\hline Drug Resistance & M. tuberculosis & Sellale & Wolaita & Nekemte \\
\hline Fully Susceptible & $14(48.3 \%)$ & $8(27.6 \%)$ & $5(17.2 \%)$ & $1(3.4 \%)$ \\
\hline Resistance to Any Drug & $15(51.7 \%)$ & $11(37.9 \%)$ & $4(13.8 \%)$ & \\
\hline Resistance to One Drug Only & $10(34.4 \%)$ & $7(24.1 \%)$ & $3(10.3 \%)$ & \\
\hline Streptomycin & $7(24.1 \%)$ & $6(20.7 \%)$ & $1(3.4 \%)$ & \\
\hline Ethambutol & $0(0.0 \%)$ & $0(0.0 \%)$ & $0(0.0 \%)$ & \\
\hline Rifampin & $1(3.4 \%)$ & $0(0.0 \%)$ & $1(3.4 \%)$ & \\
\hline Isoniazid & $2(6.8 \%)$ & $1(3.4 \%)$ & $1(3.4 \%)$ & \\
\hline Poly Resistance & $5(17.2 \%)$ & $4(13.8 \%)$ & $1(3.4 \%)$ & \\
\hline Streptomycin \& Isoniazid & $3(10.3 \%)$ & $3(10.3 \%)$ & $0(0.0 \%)$ & \\
\hline Streptomycin \& Rifampicin & $1(3.4 \%)$ & $1(3.4 \%)$ & $0(0.0 \%)$ & \\
\hline Streptomycin, Ethambutol \& Isoniazid & $1(3.4 \%)$ & $0(0.0 \%)$ & $1(3.4 \%)$ & \\
\hline
\end{tabular}


since the beginning of TB chemotherapy as of 1943 [29]. Furthermore, it is widely used to treat infectious diseases other than TB. Hence, high rate of resistance to streptomycin is expected. The relative ineffectiveness of streptomycin, route of administration, and the low level of resistance to ethambutol justify the most recent replacement of streptomycin by ethambutol by the Ethiopian National TB Program. However, the drug is still in use in the treatment of different types of TB cases [30]. Therefore, follow up of resistance to this long-aged antiTB drug requires due attention.

Next to streptomycin, the rate of resistance to isoniazid was higher (20.6\%) than the rest two drugs: ethambutol and rifampicin in the current study. Previous studies in Ethiopia showed that the frequency of resistance to isoniazid ranges from $4.1 \%$ to $21 \%$ [6] [7] [31]. An alarmingly high rate of resistance (76.03\%) to isoniazid was reported recently from Bangladesh [28].

A resistance rate of $6.8 \%$ to rifampicin observed in the present study is higher than the previous reports, $1.4 \%$ [7] and 2.5\% [31] in the country and not very different from that reported from Tanzania, 4.5\% [32]. In the present study, compared to streptomycin and isoniazid, rifampicin has still low resistance rate in spite of its indiscriminate use for other bacterial infections. Although, rifampicin resistance occurs most often in strains that are also resistant to isoniazid, no simultaneous resistance to isoniazid and rifampcin was observed in this study. It has been noted that mono resistance to isoniazid is common but monoresistance to rifampicin is quite rare [33]-[35]. Moreover, detection of polyresistance in the present study indicates the chance of occurrence of MDR-TB. Hence, detection of resistance to rifampicin should be considered seriously because it may accompany resistance to isoniazid, which is very notorious if occurs simultaneously.

In this study, no MDR-TB cases were detected. However, earlier studies in Ethiopia have reported the presence of MDR-TB in $1.2 \%$ of new and up to $12 \%$ of the re-treatment cases [30] [35]. The possible explanation of not detecting MDR-TB can be due to the small number of study isolates used in the current study. The overall low level of MDR-TB reports from the country can be due to the lack of frequent surveillance and inadequate facilities for susceptibility testing.

On the other hand, although the emergence and trans-continental spread of MDR M. bovis was evidenced [17], both mono-, poly- and MDR M. bovis was not detected. This is probably due to absence of treatment pressure in cattle population used in the present study. However, this does not guarantee the absence of transmission of drug resistant TB from humans to cattle, as $M$. tuberculosis has been already isolated from cattle in previous studies [12] [14] [36]. If cattle acquire drug resistant TB from humans, they in turn can transmit it to those at risk. The use of anti-TB drugs for prophylactic or therapeutic purposes for TB and other infectious diseases in human than in cattle leads to selective pressure favoring spread of drug resistant bacilli in humans than in cattle.

\section{Limitation of the Study}

The study may not ideally represent the general population. However, a finding based on representative study isolates does not change the fact that there is drug resistance in M. tuberculosis in the sites from where the isolates were obtained.

\section{Conclusion}

Anti-TB drug resistance to streptomycin, isonaizid, rifampicin and ethambutol is higher in M. tuberculosis isolates. Prevalence of streptomycin drug resistance is higher than that of rifampicin, ethambutol, and isoniazid among M. tuberculosis isolates. The present absence of drug resistance in $M$. bovis isolates does not mean that there is no drug resistant $M$. bovis in the sites from where the isolates were obtained. A large scale study is required to confirm the presence or absence of drug resistance in M. bovis.

\section{Acknowledgements}

The authors thank Addis Ababa University for financially supporting this study. We would also like to thank Mr. Adane Bekele and Mrs. Feven Girmachew for their technical assistance during laboratory work.

\section{References}

[1] Paramasivan, C.N. (1998) An Overview on Drug Resistant Tuberculosis in India. Indian Journal of Tuberculosis, 45, 73-81. 
[2] Rosha, D. and Kataria, V.K. (2001) Impact of Initial Drug Resistance Pattern on the Maintenance Phase of Short Course Chemotherapy with Reference to the Emergence of Multi-Drug Resistance. Indian Journal of Tuberculosis, 48, 205-207.

[3] Surucuoglu, S., Ozkutuk, N., Celik, P., Gazi, H., Dinc, G., Kurutepe, S., Koroglu, G., Havlucu, Y. and Tuncay, G. (2005) Drug-Resistant Pulmonary Tuberculosis in Western Turkey: Prevalence, Clinical Characteristics and Treatment Outcome. Annals of Saudi Medicine, 25, 313-318.

[4] Amor, Y.B., Nemser, B., Singh, A., Sankin, A. and Schluger, N. (2008) Underreported Threat of Multidrug-Resistant Tuberculosis in Africa. Emerging Infectious Diseases, 14, 1345-1352. http://dx.doi.org/10.3201/eid1409.061524

[5] Mitike, G., Kebede, D. and Yeneneh, H. (1997) Prevalence of Anti-Tuberculosis Drug Resistance in Harar Tuberculosis Centre, Ethiopia. East African Medical Journal, 74, 158-161.

[6] Gebeyehu, M., Lemma, E. and Eyob, G. (2001) Prevalence of Drug Resistant Tuberculosis in Arsi Zone, Ethiopia. Ethiopian Journal of Health Development, 15, 11-16. http://dx.doi.org/10.4314/ejhd.v15i1.9891

[7] Desta, K., Asrat, D., Lemma, E., Gebeyehu, M. and Bekele, B. (2008) Drug Susceptibility of Mycobacterium tuberculosis Isolates form Smear Negative Pulmonary Tuberculosis Patients, Addis Ababa. Ethiopian Journal of Health Development, 22, 212-215.

[8] García, J.M.G. (2006) Meat as a Vector of Transmission of Bovine Tuberculosis to Humans in Spain: A Historical Perspective. Veterinary Heritage, 29, 25-27.

[9] Signorini, L., Matteelli, A. and Bombana, E. (1998) Tuberculosis Due to Drug-Resistant Mycobaterium bovis in Pregnancy. International Journal of Tuberculosis and Lung Disease, 2, 342-343.

[10] Cosivi, O., Grange, J.M., Daborn, C.J., Raviglione, M.C., Fujikura, T., Cousins, D., Robinson, R.A., Huchzermeyer, H.F., de Kantor, I. and Meslin, F.X. (1998) Zoonotic Tuberculosis Due to Mycobacterium bovis in Developing Countries. Emerging Infectious Diseases, 4, 59-70. http://dx.doi.org/10.3201/eid0401.980108

[11] Shitaye, J.E., Tsegaye, W. and Pavlik, I. (2007) Bovine Tuberculosis Infection in Animal and Human Populations in Ethiopia: A Review. Veterinarni Medicina, 52, 317-332.

[12] Ameni, G. and Erkihun, A. (2007) Bovine Tuberculosis on Small-Scale Dairy Farms in Adama Town, Central Ethiopia, and Farmer Awareness of the Disease. Revue Scientifique et Technique de L'Office International des Epizooties, 26, 711-719.

[13] Yimenu, D. and Demissie, A. (2008) The Role of Bovine Tuberculosis as Zoonotic Disease in Ethiopia. Ethiopian Journal of Health Development, 22, 97-147.

[14] Berg, S., Firdessa, R., Habtamu, M., Gadisa, E., Mengistu, A., Yamuah, L., Ameni, G., Vordermeier, M., Robertson, B.D., Smith, N.H., Engers, E., Young, D., Hewinson, R.G., Aseffa, A. and Gordon, S.V. (2009) The Burden of Mycobacterial Disease in Ethiopian Cattle: Implications for Public Health. PLoS ONE, 4, 5068.

[15] Ameni, G., Desta, F. and Firdessa, R. (2010) Molecular Typing of Mycobacterium bovis Isolated from Tuberculosis Lesions of Cattle in Northeastern Ethiopia. Veternary Record, 167, 138-141. http://dx.doi.org/10.1136/vr.b4881

[16] Cobo, J., Asensio, A. and Moreno, S. (2001) Risk Factors for Nosocomial Transmission of Multidrug-Resistant Tuberculosis Due to Mycobacterium bovis among HIV-Infected Patients. International Journal of Tuberculosis and Lung Disease, 5, 413-418.

[17] Long, R., Nobert, E., Chomyc, S., van Embden, J., Mcnamee, C., Duran, R.R., Talbot, J. and Fanning, A. (1999) Transcontinental Spread of Multidrug-Resistant Mycobacterium bovis. American Journal of Respiratory and Critical Care Medicine, 159, 2014-2017. http://dx.doi.org/10.1164/ajrccm.159.6.9809076

[18] Guerrero, A., Cobo, J., Fortün, J., Navas, E., Quereda, C., Asensio, A., Cañón, J., Blazquez, J. and Gómez-Mampaso, E. (1997) Nosocomial Transmission of Mycobacterium bovis Resistant to 11 Drugs in People with Advanced HIV-1 Infection. The Lancet, 350, 1738-1742. http://dx.doi.org/10.1016/S0140-6736(97)07567-3

[19] Poojary, A., Nataraj, G., Metha, P. and Baveja, S. (2006) Rapid Antibiotic Susceptibility Testing of Mycobacterium tuberculosis: Its Utility in Resource Poor Settings. Indian Journal of Medical Microbiology, 24, 268-272. http://dx.doi.org/10.4103/0255-0857.29385

[20] Parreiras, P.M., Lobato, F.C.F., Alencar, A.P., de Figueiredo, T., Gomes, H.M., Boéchat, N., Lage, A.P., Assis, R.A., Pereira, M.A., Souza, P.R., Mota, P.M. and Suffys, P.N. (2004) Drug Susceptibility of Brazilian Strains of Mycobacterium bovis Using Traditional and Molecular Techniques. Memórias do Instituto Oswaldo Cruz, 99, 749-752. http://dx.doi.org/10.1590/S0074-02762004000700015

[21] Wolde Meskel, D., Abate, G., Lakew, M., Goshu, S., Selassie, A., Miorner, H. and Assefa, A. (2005) Evaluation of a Direct Colorimetric Assay for Rapid Detection of Rifampicin Resistant Mycobacterium tuberculosis. Ethiopian Journal of Health Development, 19, 51-54.

[22] Shen, X., Shen, G., Wu, J., Gui, X., Li, X., Mei, J., DeRiemer, K. and Gao, Q. (2007) Association between embB Co- 
don 306 Mutations and Drug Resistance in Mycobacterium tuberculosis. Antimicrobial Agents and Chemotherapy, 51, 2618-2620. http://dx.doi.org/10.1128/AAC.01516-06

[23] Guo, J., Xiang, W., Zhao, Q., Yang, Z. and Sun, Q. (2008) Molecular Characterization of Drug-Resistant M. tuberculosis Isolates from Sichuan Province in China. Japanese Journal of Infectious Diseases, 61, 264-268.

[24] Khanum, T., Hayat, A., Rasool, A., Hafiz, S., Azmat, R. and Talat, R. (2005) Mycobacterium Group: Identification and Sensitivities of Clinical Isolates Against First Line Drugs. Pakistan Journal of Biological Sciences, 8, 1794-1797. http://dx.doi.org/10.3923/pjbs.2005.1794.1797

[25] World Health Organization (2004) TB/HIV: A Clinical Manual. 2nd Edition, WHO/HTM/TB/2004.329, WHO, Geneva.

[26] Canetti, G., Fox, W., Khomenko, A., Mahler, H.T., Menon, N.K., Mitchison, D.A., Rist, N. and Smelev, N.A. (1969) Advances in Techniques of Testing Mycobacterial Drug Sensitivity and Use of Sensitivity Tests in Tuberculosis Control Programmes. Bulletin of World Health Organization, 41, 21-43.

[27] World Health Organization (2003) Guidelines for Surveillance of Drug Resistance in Tuberculosis. 2nd Edition, WHO, Geneva.

[28] Rahman, M., Kamal, S.M.M., Mohammed, F.R., Alam, B. and Ahasan, H. (2009) Anti-Tuberculosis Drug Resistance Pattern among Different Category of Tuberculosis Patients. Journal of Medicine, 10, 45-47. http://dx.doi.org/10.3329/jom.v10i2.2812

[29] Amukoye, E. (2008) Multi-Drug Resistant Tuberculosis: A Challenge in the Management of Tuberculosis. African Journal of Health Sciences, 15, 6-13. http://dx.doi.org/10.4314/ajhs.v15i1.30873

[30] The Ethiopian Federal Ministry of Health (FMOH) (2008) Tuberculosis, Leprosy and TB/HIV Prevention and Control Programme Manual. 4th Edition, The Ethiopian Federal Ministry of Health.

[31] Bruchfeld, J., Aderaye, G., Palme, I.B., Bjorvatn, B., Ghebremichael, S., Hoffner, S. and Lindquist, L. (2002) Molecular Epidemiology and Drug Resistance of Mycobacterium tuberculosis Isolates from Ethiopian Pulmonary Tuberculosis Patients with and without Human Immunodeficiency Virus Infection. Journal of Clinical Microbiology, 40, 16361643. http://dx.doi.org/10.1128/JCM.40.5.1636-1643.2002

[32] Kibiki, G.S., Mulder, B., Dolmans, W.M.V., de Beer, J.L., Boeree, M., Sam, N., van Soolingen, D., Sola, C. and van der Zanden, A.G.M. (2007) M. tuberculosis Genotypic Diversity and Drug Susceptibility Pattern in HIV-Infected and Non-HIV-Infected Patients in Northern Tanzania. BMC Microbiology, 7, 1471-2180. http://dx.doi.org/10.1186/1471-2180-7-51

[33] Somoskovi, A., Parsons, L.M. and Salfinger, M. (2001) The Molecular Basis of Resistance to Isoniazid, Rifampin, and Pyrazinamide in Mycobacterium tuberculosis. Respiratory Research, 2, 164-168. http://dx.doi.org/10.1186/rr54

[34] Johnson, R., Jordan, A.M., Warren, R., Bosman, M., Young, D., Nagy, J.N., Wain, J.R., van Helden, P.D. and Victor, T.C. (2008) Drug Susceptibility Testing Using Molecular Techniques Can Enhance Tuberculosis Diagnosis. Journal of Infection in Developing Countries, 2, 40-45.

[35] Abate, G. (2002) Drug-Resistant Tuberculosis in Ethiopia: Problem Scenarios and Recommendation. Ethiopian Medical Journal, 40, 79-86.

[36] Ameni, G., Aseffa, A., Engers, H., Young, D., Gordon, S., Hewinson, G. and Vordermeier, M. (2007) High Prevalence and Increased Severity of Pathology of Bovine Tuberculosis in Holsteins Compared to Zebu Breeds under Field Cattle Husbandry in Central Ethiopia. Clinical and Vaccine Immunology, 14, 1356-1361.

http://dx.doi.org/10.1128/CVI.00205-07 
Scientific Research Publishing (SCIRP) is one of the largest Open Access journal publishers. It is currently publishing more than 200 open access, online, peer-reviewed journals covering a wide range of academic disciplines. SCIRP serves the worldwide academic communities and contributes to the progress and application of science with its publication.

Other selected journals from SCIRP are listed as below. Submit your manuscript to us via either submit@scirp.org or Online Submission Portal.
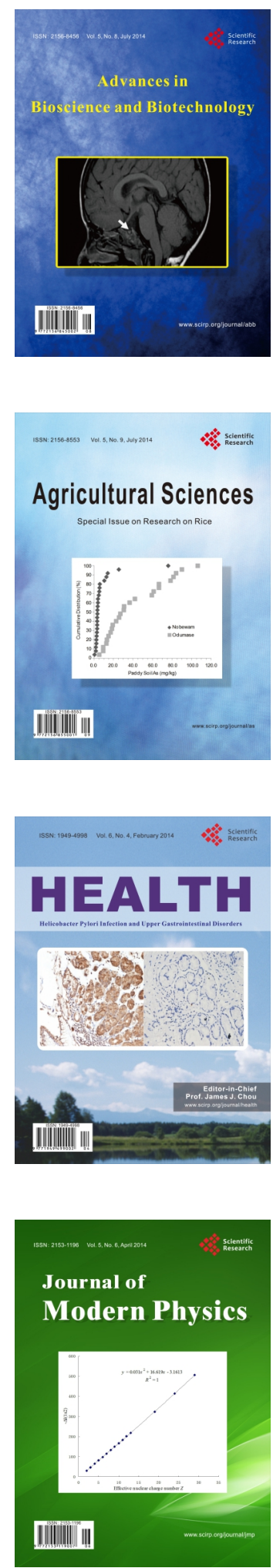
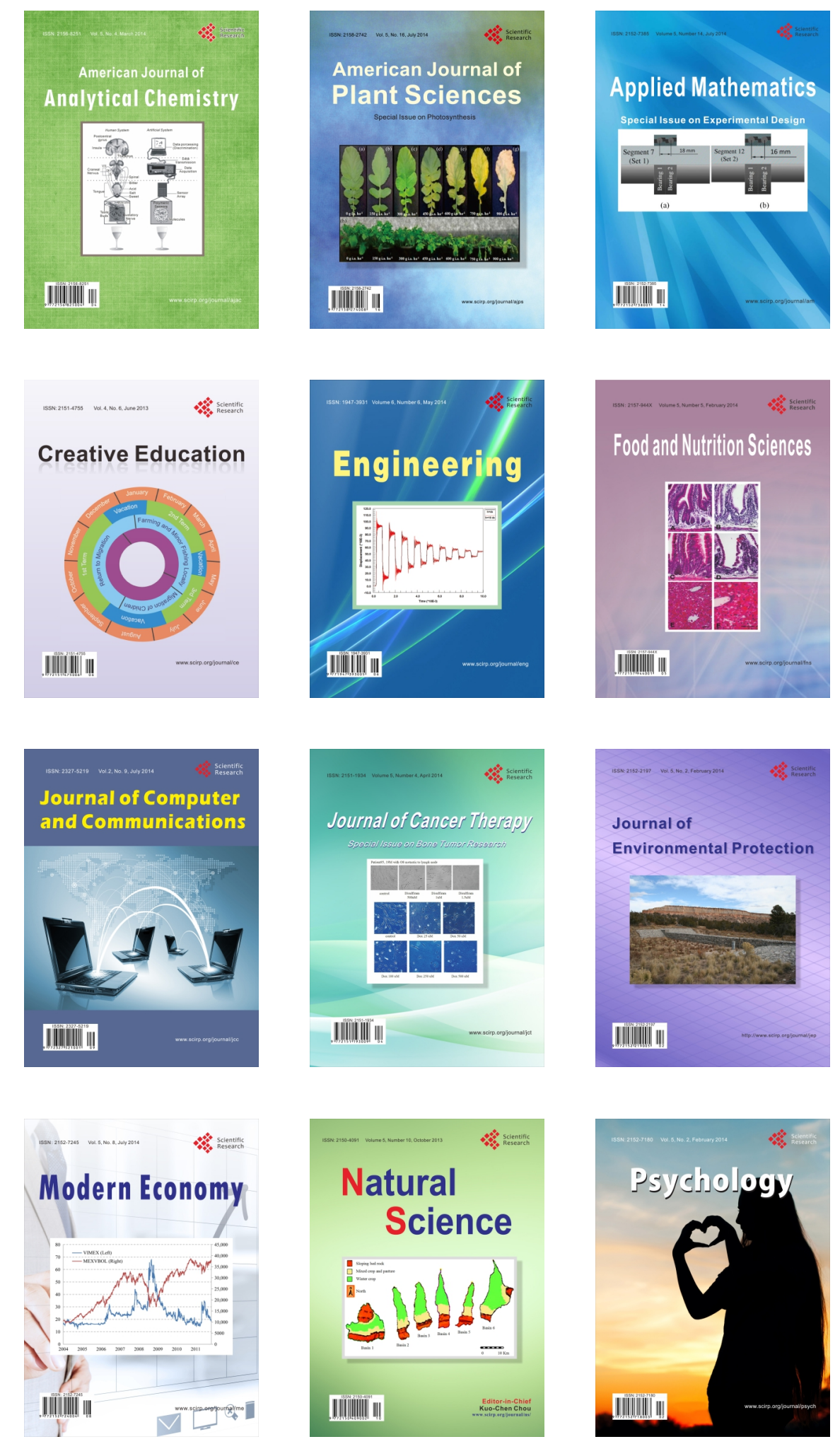